\title{
The usefulness of a new TOBEC instrument (ACAN) for investigating body composition in small mammals
}

\author{
Paweł KOTEJA
}

\begin{abstract}
Koteja P. 1996. The usefulness of a new TOBEC instrument (ACAN) for investigating body composition in small mammals. Acta Theriologica 41: 107-112.

Estimates of water mass, lean body mass (LBM) and fat content based on total body electrical conductivity (TOBEC) measured with ACAN-2 were compared with direct measurements of carcass composition in two species of rodents: the laboratory mouse $(n=20)$ and the bank vole $(n=14)$. There was a strong linear relation between ACAN readings and lean mass or water mass. Body temperature did not affected the results, but the readings were dependent on the species and sex of the individuals. That could be due to differences in the body shape. The accuracy of predicting an average LBM or water mass was very high $(1.5 \%)$, but the errors for predicting individual values were larger $(8.5 \%)$. The predicted fat content values have identical confidence intervals as that for LBM. However, the relative error for the prediction of the amount of fat was higher, because the mass of fat was much smaller than LBM.

Department of Ecosystem Studies, Institute of Environmental Biology, Jagiellonian University, Ingardena 6, 30-060 Kraków, Poland; E-mail: koteja@eko.eko.uj.edu.pl
\end{abstract}

Key words: energetics, fat, non-invasive methods, rodents

\section{Introduction}

Changes in water content and the dynamics of fat reserves are important information in physiological, bioenergetic and ecological studies of mammals. Many lines of research, both in the field and in the laboratory, require repeated measurements of gross body composition over long periods of time. However, most of the precise methods are destructive or invasive; observing changes in body composition through time requires sacrificing many animals. This is undesirable, or even impossible if the species under study is rare or protected. On the other hand, the non-invasive methods used so far are often inaccurate or difficult to use, especially in field studies.

Measurement of Total Body Electrical Conductivity (TOBEC) seems the optimum solution (Walsberg 1988, Castro et al. 1990, Anonym 1991, Scott et al. 1991, Froncisz et al. 1994, Piasecki et al. 1995). The method is entirely non-invasive. The measurement takes only a few seconds and may be repeated frequently in the same individual, providing relatively high accuracy.

So far TOBEC analyzers in eco-physiological research have been used mostly with birds. The results have been promising, although the usefulness of the 
instruments manufactured so far has been questioned in intraspecific studies on small animals (Walsberg 1988, Castro et al. 1990, Anonym 1991, Morton et al. 1991, Roby 1991, Scott et al. 1991).

The new instrument for Animal-body Composition Analysis (ACAN) has been built by Jagmar (Kraków, Poland) in collaboration with the Department of Biophysics, Jagiellonian University. The unique design of the measurement chamber allows an extremely uniform electromagnetic field to be generated. Therefore, the results are less affected by the position of the object examined and an improved accuracy is expected (Froncisz et al. 1994, Piasecki et al. 1995).

The aim of this study was to evaluate the usefulness of the new instrument for the studies on intraspecific variation of lean body mass, water and fat content in small mammals.

\section{Material and methods}

Calibration was performed with an ACAN-2 prototype (Jagmar, Poland) with the measuring chamber of diameter $46 \mathrm{~mm}$ and length $100 \mathrm{~mm}$. Two small rodent species were used: the laboratory mouse Mus musculus Linnaeus, 1758 (9 males, 11 females) and the bank vole Clethrionomys glareolus Schreber, 1780 (6 males, 8 females).

Body temperature (TB) of the animals was measured with a fine rectal probe to the nearest $0.1^{\circ} \mathrm{C}$ (thermometer: Albhorn Therm 2244-1, probe: NTC type C 856-1). Then the animals were placed into a cylindrical container (diameter $32 \mathrm{~mm}$, length $85 \mathrm{~mm}$ ) and weighed (to the nearest $0.1 \mathrm{~g}$ ). They were not anaesthetized and could change slightly their position in the chamber. For each individual 6-8 readings were taken; minimum and maximum readings were rejected before calculation of an average value.

After taking the ACAN readings the animals were killed and dried to constant mass in a vacuum drier at $60^{\circ} \mathrm{C}$. The amount of lipids in the dry mass was determined by ethyl ether extraction in a Soxhlet apparatus (Sawicka-Kapusta 1975). The analysis was performed on whole bodies, including gut contents. Lean body mass (LBM) and water mass (WM) were calculated by subtracting fat mass and dry mass, respectively, from total body mass.

Relationship between the ACAN readings (AR, dependent variable) and LBM or WM, body temperature, sex and species (independent variables) was examined using analysis of covariance (ANCOVA). After evaluating the proper models, predictive formulas for LBM and WM from ACAN measurement were obtained by rearranging the ANCOVA regression equations. The prediction accuracy of LBM and WM from the ACAN measurements was assessed employing a "jackknife" resampling procedure (Sokal and Rohlf 1981). The ANCOVA regression equation was calculated from a sample containing all but one individual. Then the actual (measured) LBM (or WM) of this individual was compared with the values estimated using the equation, and the individual's ACAN reading. Thus, the prediction was based on the calibration equation independent of the individual's data. The procedure was repeated for all individuals and then statistics for prediction errors was computed. All the computations were performed with SYSTAT (Wilkinson 1986).

\section{Results}

The relation between ACAN readings and LBM or WM was linear and highly significant: the simple regression coefficients of determination were 0.92 for LBM, 
and 0.95 for WM $(p<0.001)$. Preliminary ANCOVA showed that interactions between the covariates (TB, LBM or WM) and the categorical variables (species, sex) were not significant (the slopes of the regressions did not differ between the species or between the sexes). ANCOVA without that interactions indicated that the effect of body temperature was not significant, either. However, it revealed a statistically significant effect of species and sex on the relation between AR and LBM: with the same LBM, the mice had higher AR than the voles, and males had lower AR than females $(p<0.001$, Fig. 1 , Table 1$)$. The same was true with the

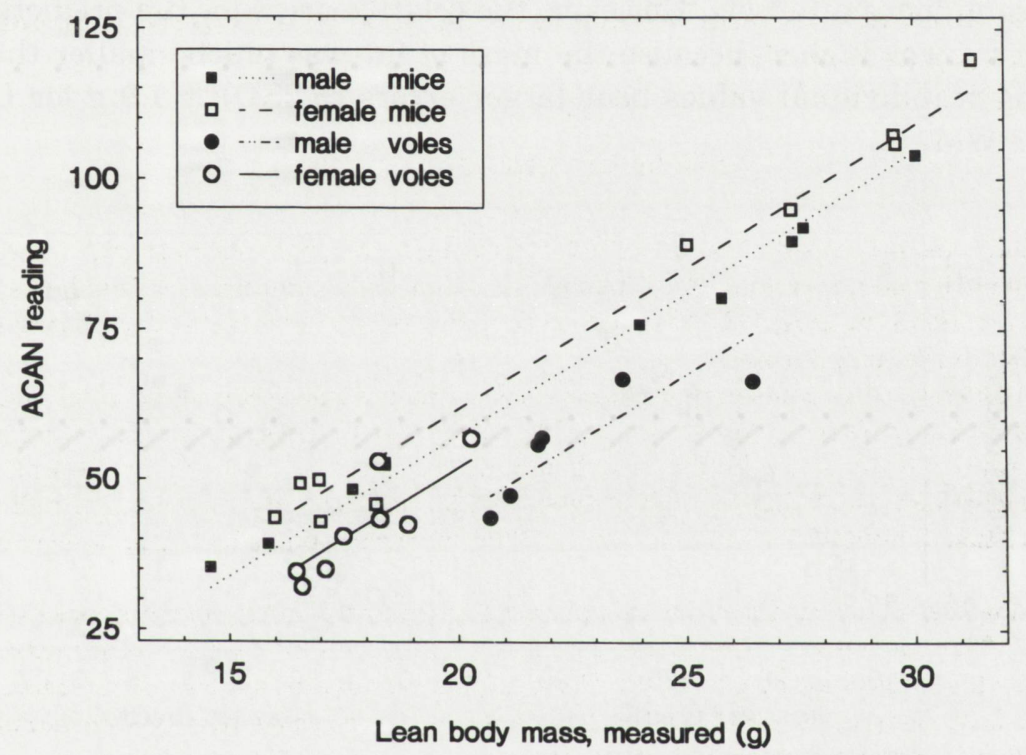

Fig. 1. Relation between ACAN readings and lean body mass in 20 laboratory mice and 14 bank voles.

Table 1. The effects of species and sex on the relation between (A) lean body mass and ACAN readings $\left(R^{2}=0.980\right)$ and $(\mathrm{B})$ body water mass and ACAN readings $\left(R^{2}=0.984\right)$. Coding of the effects: species $=1$ for mice, -1 for voles; sex $=1$ for males, -1 for females.

\begin{tabular}{|c|c|c|c|c|c|c|c|}
\hline \multirow{2}{*}{ Effect } & \multicolumn{3}{|c|}{ A } & \multirow{2}{*}{ Effect } & \multicolumn{3}{|c|}{ B } \\
\hline & Coefficient & $\mathrm{SE}$ & $p$ & & Coefficient & $\mathrm{SE}$ & $p$ \\
\hline Constant & -40.5 & 3.3 & 0.000 & Constant & -38.4 & 2.9 & 0.000 \\
\hline LBM & 4.7 & 0.1 & 0.000 & WM & 6.4 & 0.2 & 0.000 \\
\hline Species & 5.8 & 0.7 & 0.000 & Species & 4.2 & 0.7 & 0.000 \\
\hline Sex & -3.1 & 0.7 & 0.000 & Sex & -2.4 & 0.7 & 0.001 \\
\hline Species $\times$ sex & 0.9 & 0.7 & 0.223 & Species $\times$ sex & 1.2 & 0.6 & 0.076 \\
\hline
\end{tabular}


body water mass. Thus, predictive equations could be constructed from the ANCOVA regression coefficients including species and sex effects (Table 1):

$$
\begin{aligned}
& \mathrm{LBM}=(\mathrm{AR}+40.5-5.8 \mathrm{G}+3.1 \mathrm{~S}-0.9 \mathrm{GS}) / 4.7 \\
& \mathrm{WM}=(\mathrm{AR}+38.4-4.2 \mathrm{G}+2.4 \mathrm{~S}-1.2 \mathrm{GS}) / 6.4
\end{aligned}
$$

where $S=1$ for males, $S=-1$ for females, and $G=1$ for mice, $G=-1$ for voles.

The correlations between ACAN-predicted and measured directly values of LBM, WM and fat content were very high (Table 2, Fig. 2). The $95 \%$ confidence intervals were $0.33 \mathrm{~g}$ for predicted mean LBM and $0.22 \mathrm{~g}$ for predicted mean WM (Table 2). Assuming that the total body mass is determined without error, the predicted fat content values have an identical distribution of errors as LBM, and identical confidence intervals. However, the relative error for the prediction of the amount of fat was higher, because the mass of fat was much smaller than LBM. Predictions of individual values bear larger errors $( \pm 2 \mathrm{SD}): \pm 1.9 \mathrm{~g}$ for $\mathrm{LBM}$ and $\pm 1.3 \mathrm{~g}$ for WM.
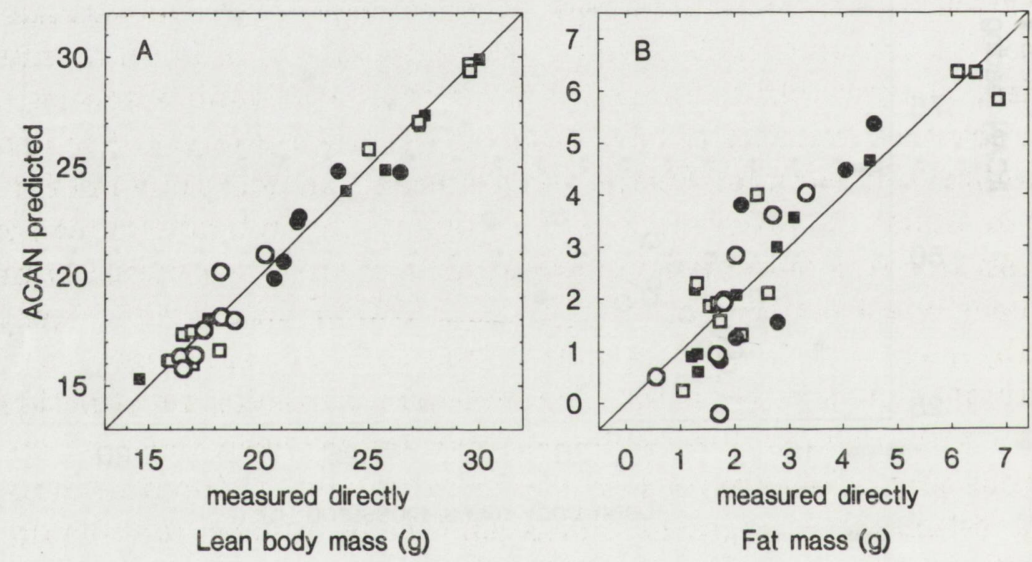

Fig. 2. ACAN predicted vs directly measured lean body mass (A) and fat mass (B) in laboratory mice and bank voles. The solid lines indicate perfect fit. Symbols are as in Fig. 1.

Table 2. Statistics for prediction errors (= difference between a value predicted with ACAN and the one measured directly) based on jackknife resampling procedure: $95 \%$ confidence interval for predicted average value $\left(=t_{0.95} \times \mathrm{SE}\right.$ and $95 \%$ confidence interval for predicted individual value $(=2 \times \mathrm{SD})$. LBM lean body mass, FM - fat mass, WM - body water mass.

\begin{tabular}{lccc}
\hline & LBM & FM & WM \\
\hline Average value (g) & 21.8 & 2.7 & 15.8 \\
$95 \%$ CI for average (g) & 0.3 & 0.3 & 0.2 \\
\% of average & 1.5 & 12.2 & 1.4 \\
$95 \%$ CI for individual (g) & 1.9 & 1.9 & 1.3 \\
\% of average & 8.7 & 70.0 & 8.2 \\
\hline
\end{tabular}




\section{Discussion}

Principally, ACAN readings depend on the temperature of the object tested, because an electric conductivity is temperature dependent. However, for the body temperatures ranging from $35^{\circ} \mathrm{C}$ to $38.5^{\circ} \mathrm{C}$ the effect was not significant. On the other hand, the readings were different in mice and voles with the same LBM or WM. Additionally, the readings were also different in males and females. Similar effect has been found in Apodemus tested with ACAN-1 prototype (Froncisz et al. 1994). That could be due to different shape of the animals, or different distribution of fat and lean tissue within their bodies. Such effects are inherent in the TOBEC method: although the electromagnetic field is uniform within an empty ACAN's resonant chamber, it is not uniform within an animal body. Therefore, with the same LBM, animals with a larger diameter will give a higher response (Piasecki et al. 1995).

The differences between the species and the sexes affected the offsets, rather than the slopes of the calibration equations (at least within that range of LBM). If the objective of a study is to follow relative changes of body composition it may be sufficient to use a calibration equation obtained in a similar species. However, when the absolute amounts of lean tissue or fat are of interest, it is necessary to obtain a calibration equation for the given species, including any information that will help to adjust the differences in the shape of the individuals (eg sex, age).

The instrument is suitable for many studies of variation in body composition. The accuracy of estimating an average LBM, WM or fat content is very high, as indicated by narrow confidence intervals (Table 2, Fig. 2). However, the predictions for individuals bear a larger error. The instrument enables a distinction between individuals with high and low fat content, but not between those which have one or two grams of fat. This error is partly due to individual differences in body shape. Thus, the estimated changes of individuals' body composition should be more accurate (Oklejewicz and Koteja 1995). To improve the accuracy, the animals should be placed into a holder forcing a constant diameter.

Acknowledgements: I wish to thank Jagmar company (ACAN producer) for providing the instrument and for supporting my participation in the II European Congress of Mammalogy in Southampton. The study was partly supported by the Committee for Scientific Research grant KBN 4-1546-91-01 to J. Weiner. I am also grateful for his comments on the manuscript.

\section{References}

Anonym 1991. EM-SCAN Inc. Model SA 2 Small research Animal Body Composition Analyzer. Operation Manual. Em-Scan Inc., Springfield, IL: 1-63.

Castro G., Wunder B. A. and Knopf F. L. 1990. Total body electrical conductivity (TOBEC) to estimate total body fat of free-living birds. Condor 92: 496-499.

Froncisz W., Piasecki W., Koteja P., Staliński J. and Weiner J. 1994. A new instrument for non-invasive measurement of total body water and fat content in small mammals. Polish Ecological Studies 20: $323-328$. 
Morton J. M., Kirkpatrick R. L. and Smith E. P. 1991. Comments on estimating total body lipids from measurements of lean body mass. Condor 94: 463-465.

Oklejewicz M and Koteja P. 1995. Energy and fat balance in laboratory mice: a study with a new TOBEC instrument. Abstracts of II European Congress of Mammalogy, 27.03-1.04.1995, Southampton, England: 76.

Piasecki W., Koteja P., Froncisz W. and Weiner J. 1995. New way of body composition analysis using total body electrical conductivity method. Review of Scientific Instrument 66(4): 3037-3041.

Roby D. D. 1991. A comparison of two noninvasive techniques to measure total body lipids in live birds. Auk 108: 509-518.

Sawicka-Kapusta K. 1975. Fat extraction in the Soxhlet apparatus. [In: Methods for ecological bioenergetics. W. Grodziński, R. Z. Klekowski and A. Duncan, eds]. Blackwell Scientific Publications, Oxford: 288-292.

Scott I., Grant M. and Evans P. R. 1991. Estimation of fat-free mass of live birds: use of total body electrical conductivity (TOBEC) measurements in studies of single species in the field. Functional Ecology 5: 314-320.

Sokal R. R. and Rohlf F. J. 1989. Biometrics. 2nd edition. Freeman, New York: 1-859.

Walsberg G. E. 1988. Evaluation of a nondestructive method for determining fat scores in small birds and mammals. Physiological Zoology 61: 153-159.

Wilkinson L. 1986. SYSTAT. The system for statistics. Systat Inc., Evanston, IL.

Received 21 April 1995, accepted 2 October 1995. 